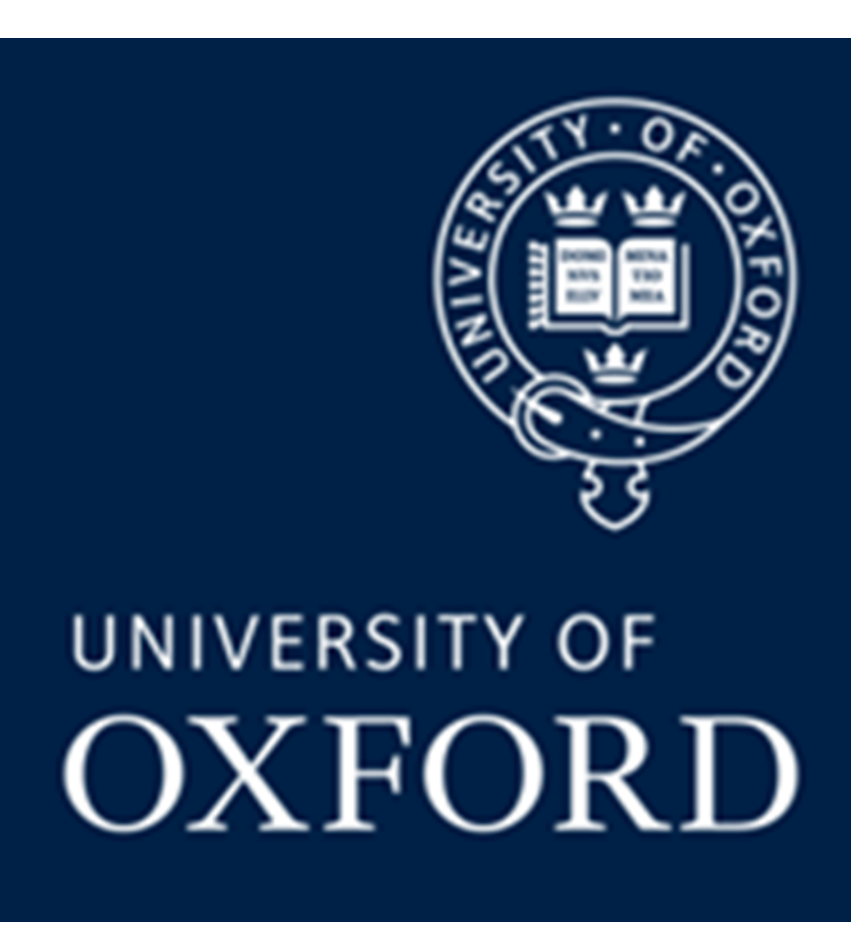

\title{
Somatosensory proffles in patients with non-specific neck-arm pain with and without positive neurodynamic tests
}

\section{Department of Physiotherapy, Sir Charles Gairdner Hospital, Perth, Western Australia
${ }^{3}$ School of Physiotherapy and Exercise Science, Curtin University, Perth, Western Australia \\ ${ }^{4}$ Faculty of Business Management and Social Sciences, Hochschule Osnabrück, University of Applied Sciences,}

Karina Boettger ${ }^{1}$, Brigitte Tampin²,3,4, Nikolaus Ballenberger ${ }^{4}$, Landmann Gunther ${ }^{1}$, Lenka Stockinger ${ }^{1}$, Annina Schmid

\section{Background}

While clinical neurological examination and electrodiagnostic studies are usually normal in patients with non-specific neck arm pain (NSNAP), a subgroup of patients presents with clinical signs of whe

\section{Purpose}

The aim of this study was to evaluate potentho patients with u

\section{Methods}

Forty patients with unilateral NSNAP participated in the study. All patients had normal electrodiagnostic tests. Standardised upper limb neurodynamic tests were used to divide patients into those with heightened nerve mechanosensitivity (ULNT ${ }_{\text {Pos) }}$ ) and those with normal nerve mechanosensitivity (ULNT NEG). Quantitative sensory testing was performed according to the protoco patients' maximal pain area. Corresponding areas were measured in 26 healthy controls.

\section{Results}

Demographic and clinical characteristics of patients and healthy controls are documented in Table

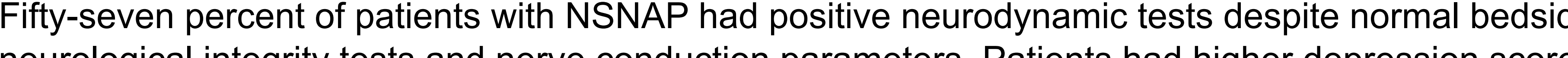
neurological integrity tests and nerve conduction parameters. Patients had higher depression scores patients' depression scores fell within the normal range.

\begin{tabular}{|c|c|c|c|c|}
\hline & $\begin{array}{c}\mathrm{HC} \\
(n=26)\end{array}$ & $\begin{array}{l}\text { ULNTNEG } \\
(n=17)\end{array}$ & $\begin{array}{c}\text { ULNTPos } \\
(n=23)\end{array}$ & Pvalue \\
\hline & & $46.5(12.4)$ & & 0.969 \\
\hline Gender (female) & 12 & 11 & 17 & 0.129 \\
\hline Symptom duration (years) & & $6.47(4.02)$ & $5.74(3.52)$ & 0.435 \\
\hline HADS & & & & \\
\hline $\begin{array}{l}\text { Anxiety } \\
\text { (normal range } \leq 10 \text { ) } \\
\text { Depression } \\
\text { (normal range } \leq 10 \text { ) }\end{array}$ & $\begin{array}{l}4.27(4.2) \\
1.23(1.8)\end{array}$ & $\begin{array}{l}6.41(3.26) \\
5.12(3.59)\end{array}$ & $\begin{array}{l}6.69(4.45) \\
5.26(4.75)\end{array}$ & $\begin{array}{c}0.060 \\
<0.001\end{array}$ \\
\hline Sleep last week (VAS) & $2.9(2.6)$ & $3.59(2.42)$ & $4.57(2.4)$ & 0.1371 \\
\hline & & & & \\
\hline Physical & & $39.76(8.5)$ & $38.46(10.33)$ & 0.673 \\
\hline $\begin{array}{l}\text { Mental } \\
\text { NDI }\end{array}$ & & $\begin{array}{l}46.32(9.58) \\
2494(751)\end{array}$ & $46.23(11.86)$ & 0.978 \\
\hline DASH & & 4 & $57.96(20.9)$ & 0.113 \\
\hline PainDETECT & & $11.94(6.35)$ & $15.43(5.95)$ & 0.820 \\
\hline
\end{tabular}

Disabilities of the Amm, Shoulder and Anxiety and Depression Scale; NDI; Neck Disability Index; DASH,

\section{Somatosensory profiles - Detection thresholds}

Patients with ULNT pos had a significant deficiti in cold, mechanical and vibration detection compared to healthy controls $(p=0.021, p=0.002$ and $p=0.001$ respectively); patients with
ULNT subgroups had loss of function in the thermal sensory limen compared to healthy controls (p<0.008). There were no significant differences in detection thresholds between patient groups. Of note, the ANOVA linear trend analysis revealed a significant dose response relationship with the ULNT $T_{N E G}$ representing an intermediate phenotype between ULNT $T_{\text {POS }}$ patients and healthy controls for mechanical and vibration detection $(p<0.004)$.

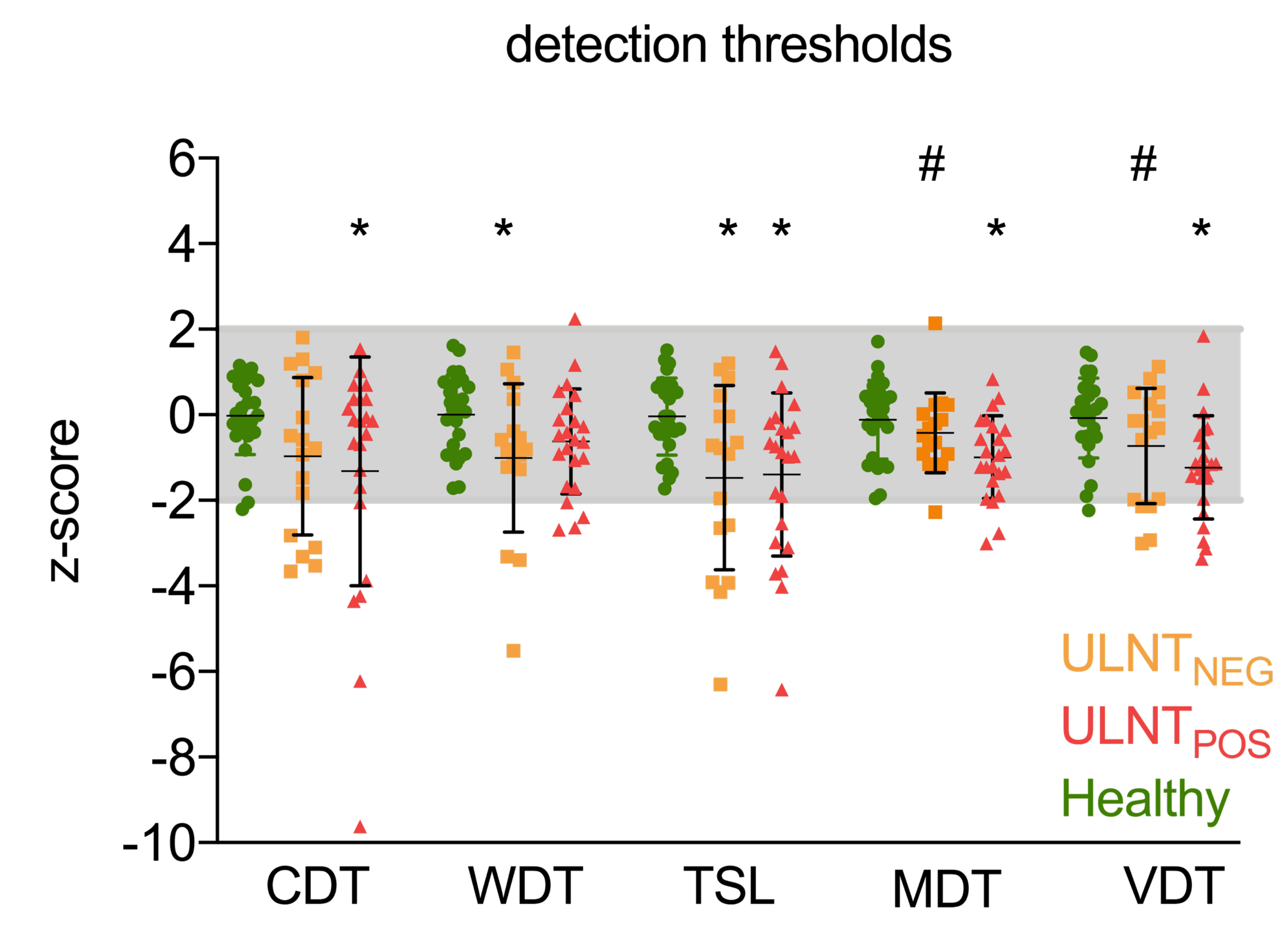

Fig.1. Sensory profiles (SEM) of CDT, cold detection threshold; WDT, warm detection
threshold; TSL, thermal sensory limen; MDT, mechanical detection threshold; VDT vibration threshold; TSL, thermal sensory limen; MDT, mechanical detection threshold; VDT vibration
detection threshold; indicates significant
\#indiference compared to healthy controls. \#indicates significance
of the ULNT

Somatosensory profiles - Pain thresholds Patients with NSNAP demonstrated significantly lower pain thresholds for cold, heat and pressure pain compared to healthy controls ( $p=0.001, p=0.002$ and $p=0.002$, Figure 2$)$. There was no difference in any sensory parameters between patient groups. The ULNT ${ }_{N E G}$ subgroup thermal and pressure pain thresholds $(p<0.024)$.

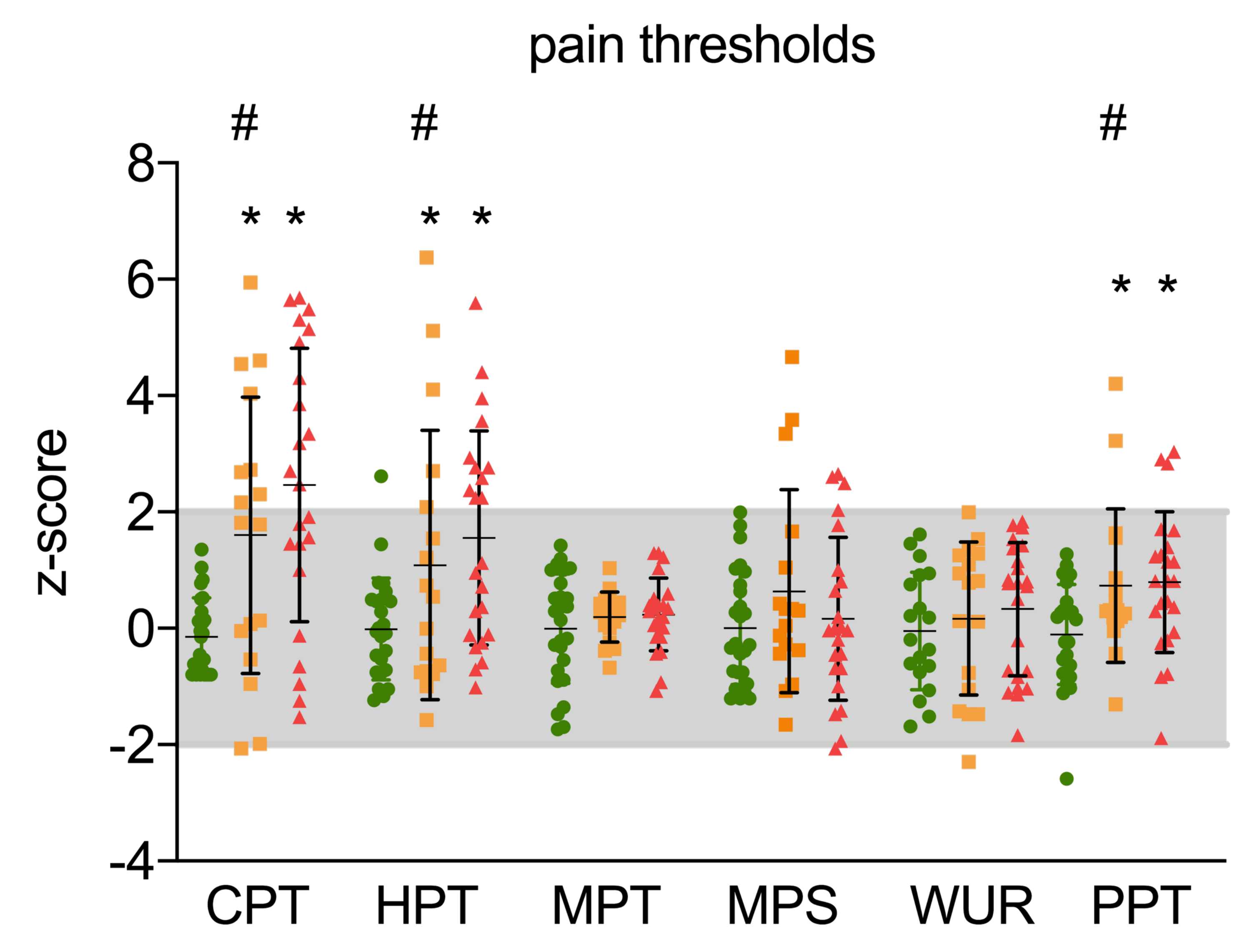

Fig.2. Sensory profiles (SEM) of CPT, cold pain threshold; HPT, heat pain threshold; MPT, mechanical pain threshold; MPS mechanical pain sensitivity; wUR, wind up ratio; PPT,
pressure pain threshold. . indicates significant difference compared to healthy controls; \# indicates significan

Conclusion

Heightened nerve mechanosensitivity was present in $57 \%$ of patients with NSNAP. Even though

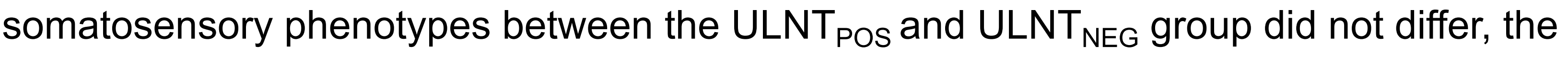
comparison to healthy controls revealed a more pronounced loss of function phenotype of the ULNT pos group. It stands to debate if this loss of function is suggestive of a subtle nerve
dysfunction or should be interpreted as a consequence of altered central pain processing

Acknowledgement The study was supported by the National Institute for Health Research (NIHR) Oxford Biomedical Research Centre Bowls Association Grant) and the Physiotherapy Research Foundation (seeding grant) Australia.

References

(1997) Physical tritation of the Reterences

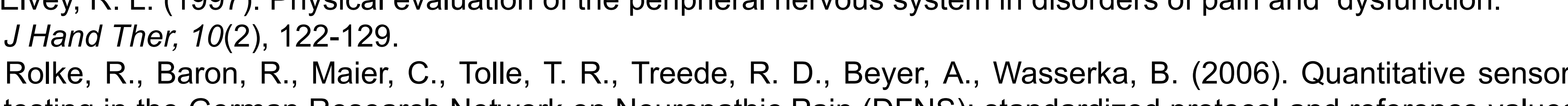

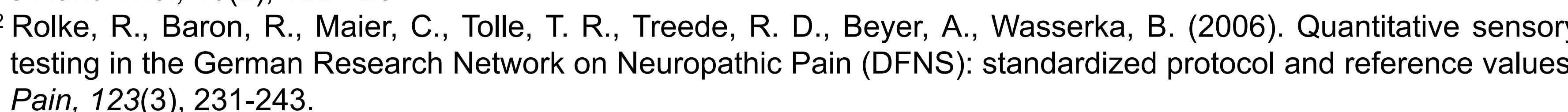
Tampin B, Slater H, Hall T, Lee G, Briffa NK.(2012). Quantitative sensory testing somatosensory profiles in patients
with cervical radiculopathy are distinct from those in patients with nonspecific neck-arm pain. Pain 153(12),2403-14. 